\title{
Caracterización de trasplantados renales con donantes de criterios expandidos
}

\section{Characterization of kidney transplant patients with expanded criteria donors}

\author{
Paola Karina García-Padilla, María José Vargas-Brochero, Maité Hurtado-Uriarte, \\ Camilo Alberto González-González, Martha Patricia Rodríguez-Sánchez, \\ Juan Agustín Patiño-Trejos, Kateir Mariel Contreras-Villamizar • Bogotá, D.C. (Colombia)
}

DOI: https://doi.org/10.36104/amc.2019.1185

\section{Resumen}

Introducción: el trasplante renal es el tratamiento de elección para pacientes con enfermedad renal crónica, sin embargo, el número de donantes es insuficiente y las listas de espera crecen exponencialmente cada año. El trasplante con donante de criterios expandidos beneficia a un número importante de pacientes, mejorando su supervivencia al compararlos con los que permanecen en diálisis. El objetivo de este estudio es describir las características de pacientes trasplantados con donantes de criterios expandidos y su función renal al primer y tercer año del trasplante.

Métodos: se realizó un estudio descriptivo observacional. Se eligieron pacientes mayores de 18 años trasplantados con donante de criterios expandidos entre 2007 y 2015.

Resultados: de un total de 227 pacientes analizados, 18 fueron trasplantados con donante de criterios expandidos. Los receptores tenían 59.5 años (37-79), tiempo en lista de espera de 22.4 meses (2.6-77.8), tiempo en diálisis de 4.5 años (0.5-18.4). La edad del donante fue 61 años (50-73). La supervivencia del injerto al año fue $88.9 \%$ y a los tres años de $80 \%$. El $11.1 \%$ de los pacientes presentaron rechazo celular agudo al año y el promedio de la tasa de filtración glomerular al primer y tercer año fue de $58.4 \mathrm{~mL} / \mathrm{min} / 1.73 \mathrm{~m}^{2}$.

Conclusión: los pacientes trasplantados con donante de criterios expandidos tienen una buena función del injerto renal a tres años, con supervivencia del injerto y del paciente, similar a la reportada con donante con criterio estándar. (Acta Med Colomb 2019; 44. DOI: https://doi.org/10.36104/ amc.2019.1185).

Palabras clave: selección del donante, supervivencia del injerto, rechazo del injerto, trasplante renal.

\footnotetext{
Abstract

Introduction: renal transplantation is the treatment of choice for patients with chronic kidney disease; however, the number of donors is insufficient and waiting lists grow exponentially every year. Transplantation with expanded criteria donors benefits a significant number of patients, improving their survival when comparing them with those who remain on dialysis. The objective of this study is to describe the characteristics of the transplanted patients with the expanded criteria donors and their renal function at the first and third year of the transplant.

Methods: an observational descriptive study was performed. Patients over 18 years of age transplanted with an expanded criteria donor between 2007 and 2015 were chosen.

Results: of a total of 227 patients analyzed, 18 were transplanted with an expanded criteria donor. Recipients were 59.5 years old (37-79), waiting list time of 22.4 months (2.6-77.8), dialysis time of 4.5 years (0.5-18.4). The donor's age was 61 years (50-73). Graft survival at one year was $88.9 \%$ and at three years was $80 \% .11 .1 \%$ of the patients presented acute cellular rejection at one year and the average glomerular filtration rate at the first and third year was $58.4 \mathrm{~mL} / \mathrm{min} / 1.73 \mathrm{~m}^{2}$.

Conclusion: Patients transplanted with an expanded criteria donor have a good function of the renal graft at three years, with graft and patient survival, similar to that reported with a donor with standard criteria. (Acta Med Colomb 2019; 44. DOI: https://doi.org/10.36104/amc.2019.1185).

Keywords: donor selection, graft survival, graft rejection, kidney transplant.
}

Dra. Paola Karina García-Padilla: Internista Nefróloga. Pontificia Universidad Javeriana, Facultad de Medicina, Hospital Universitario San Ignacio; Dras.: María José Vargas-Brochero y Maité Hurtado-Uriarte: Residentes de Nefrología, Pontificia Universidad Javeriana; Camilo Alberto González-González: Internista, Nefrólogo. Pontificia Universidad Javeriana, Facultad de Medicina, Hospital Universitario San Ignacio; Dra. Martha Patricia Rodríguez-Sánchez: Internista Nefróloga. Pontificia Universidad Javeriana, Facultad de Medicina, Hospital Universitario San Ignacio; Juan Agustín PatiñoTrejos: Estudiante de Medicina. Pontificia Universidad Javeriana, Facultad de Medicina; Dra. Kateir Mariel Contreras-Villamizar: Internista, Nefróloga. Pontificia Universidad Javeriana, Facultad de Medicina, Hospital Universitario San Ignacio. Bogotá, D.C. (Colombia). Correspondencia: Dra. Paola Karina GarcíaPadilla, Bogotá, D.C. (Colombia). E-mail: pkgarcia@husi.org.co Recibido: 11/I/2018 Aceptado: 22 /VII/2019 


\section{Introducción}

La enfermedad renal crónica (ERC) es un problema de salud pública que afecta a todas las poblaciones, creciendo de forma exponencial en los últimos años. Actualmente más de 2500000 de personas en el mundo dependen de terapias de reemplazo renal para manejo de su patología, lo cual disminuye la calidad de vida y la supervivencia y acarrea una carga económica alta para los sistemas de salud; para el 2014 correspondió a 2\% del gasto en salud en Colombia (1).

El trasplante renal es el mejor tratamiento para la ERC estado 5, permite la recuperación total de las funciones renales, mejora calidad de vida y supervivencia de los pacientes. Sin embargo, la oferta de órganos es baja, creando la necesidad de estrategias que aumenten el pool de donantes, como el uso de donantes con criterios expandidos (DCE) (2). El DCE es definido como aquel mayor de 60 años o mayor de 50 años que cumpla con dos de los siguientes tres criterios: hipertensión arterial, creatinina sérica $>1.5 \mathrm{mg} / \mathrm{dL}$ o muerte debida a enfermedad cerebrovascular (ECV) (3, 4). En este escenario, algunos de los factores que influyen en los resultados del trasplante renal, como son: la edad del donante, la presencia de comorbilidades como hipertensión arterial y diabetes mellitus (5), los fenómenos de isquemiareperfusión (6), generan preocupación por las repercusiones a corto, mediano y largo plazo sobre la funcionalidad y supervivencia del injerto. Estudios a gran escala y en diferentes localizaciones geográficas a nivel mundial, han mostrado resultados comparables en supervivencia del injerto y del paciente, entre DCE y donante con criterios estándar (DCS), principalmente en los receptores mayores de 40 años $(2,3,7)$.

En Colombia se encontraban 2607 pacientes en lista de espera para trasplante renal a diciembre de 2018 y la tasa de donación fue de 8.3 por millón de población (8), por lo que el uso de estas estrategias en nuestro país es fundamental.

\section{Material y métodos}

Se realizó un estudio observacional descriptivo. Los pacientes fueron seleccionados de acuerdo con los criterios de inclusión: mayores de 18 años con trasplante renal de DCE del 2007 al 2015. Todos los donantes tenían biopsia renal con puntaje de Remuzzi 3 (9). Aquellos pacientes en quienes no fue posible realizar seguimiento, fueron excluidos. Se realizaron análisis descriptivos de las características sociodemográficas y clínicas de la población del estudio. Se presentan las medidas de resumen para variables cualitativas en proporciones, y para las variables cuantitativas en medianas y rangos, considerando el tamaño de muestra pequeño por conveniencia.

\section{Resultados}

Se analizaron 227 pacientes trasplantados renales, $18 \mathrm{de}$ ellos trasplantados con DCE (7.9\%).

Los receptores tenían una mediana de edad de 59.5 años (37-79). Uno de los pacientes trasplantados tenía menos de 40 años, pero llevaba 12 años en diálisis, por lo que se decidió optar por un DCE. Dentro de las causas de ERC, en seis pacientes se desconocía la etiología, tres eran diabéticos, cuatro tenían enfermedad poliquística renal, dos tenían enfermedad glomerular, dos eran hipertensos y otro tenía enfermedad tubulointersticial. La mediana del tiempo en lista de espera fue de 22.4 meses (2.6-77.8) y en diálisis de 4.5 años (0.5-18.4). El 38.8\% tenían PRA (Panel reactivo de anticuerpos) mayor de 20\%. La edad del donante fue 61 años (50-73) y el tiempo de isquemia fría promedio fue de 14.5 horas (Tabla 1). El 67\% de los pacientes recibieron inducción con timoglobulina y el resto basiliximab. La principal causa de muerte fue ataque cerebrovascular en 15 de los 18 donantes.

Los resultados clínicos muestran supervivencia del injerto y de paciente de 88.9 - $80 \%$ al año, y tres años respectivamente. Las causas de mortalidad fueron de origen infeccioso, por enfermedad por citomegalovirus, histoplasmosis y sepsis bacteriana, y una de origen cardiovascular a los cinco años del trasplante.

El 11.1\% de los pacientes presentaron rechazo agudo celular al año, y el promedio de la tasa de filtración glomerular por MDRD4 al primer y tercer año fue de $58.4 \mathrm{~mL} / \mathrm{min} / 1.73 \mathrm{~m}^{2}$ (Tabla 2).

Tabla 1. Datos demográficos de los pacientes trasplantados con DCE.

\begin{tabular}{|c|c|}
\hline Variable & $\mathrm{n}=18$ \\
\hline Masculino & $11(61.1 \%)$ \\
\hline Edad del receptor en años & $59.5(37-79)$ \\
\hline \multicolumn{2}{|l|}{ Etiología de ERC } \\
\hline - Idiopática & $6(33.3 \%)$ \\
\hline - $\quad$ Enfermedad poliquística & $4(22.2 \%)$ \\
\hline - $\quad$ Diabetes mellitus & $3(16.7 \%)$ \\
\hline - Hipertensión arterial & $2(11.1 \%)$ \\
\hline - $\quad$ Glomerulonefritis & $2(11.1 \%)$ \\
\hline - $\quad$ Otras & $1(5.6 \%)$ \\
\hline Tiempo en lista (meses) & $22.4(2.6-77.8)$ \\
\hline Tiempo en diálisis (años) & $4.5(0.5-18.4)$ \\
\hline PRA mayor $20 \%$ & $7(38.8 \%)$ \\
\hline Tiempo de isquemia fría (horas) & $14.5(8-19)$ \\
\hline \multicolumn{2}{|l|}{ Inducción } \\
\hline - $\quad$ Timoglobulina & $12(66.7 \%)$ \\
\hline - $\quad$ Basiliximab & $6(33.3 \%)$ \\
\hline \multicolumn{2}{|l|}{ Datos donante } \\
\hline Edad en años & $61(50-73)$ \\
\hline Creatinina (mg/dL) & $0.75(0.6-2.1)$ \\
\hline PRA: Panel reactivo de anticuer & \\
\hline
\end{tabular}




\section{Discusión}

A pesar de los esfuerzos y cambios recientes en la legislación colombiana, la tasa de donación disminuyó durante el último año (8) y los pacientes con ERC en diálisis y lista de espera de trasplante continúan en aumento (10). La mortalidad del paciente en diálisis es elevada, con cifras que alcanzan $50 \%$ a cinco años (11), por ello la búsqueda de estrategias que aumenten la población de donantes es una necesidad.

Se presenta una serie de casos de pacientes que recibieron injertos de DCE, con óptima función del injerto a uno y tres años y baja tasa de complicaciones.

El uso de DCE, genera controversias por las mayores tasas de función retardada del injerto y falla primaria que se han reportado al compararlo con el uso de DCS. Bokzurt B y colaboradores, encontraron pérdida del injerto en $26 \%$ de casos de DCE comparado con $10 \%$ con el uso de DCS (12). Adicionalmente, estos trasplantes se pueden asociar a costos más elevados, al aumentar las probabilidades de requerir diálisis, admisiones a urgencias e incluso mayor mortalidad intraoperatoria, sin embargo, los receptores de DCE tienen $72 \%$ menos mortalidad comparado con los pacientes en lista de espera (13).

Un estudio francés multicéntrico observacional incluyó 2763 pacientes trasplantados renales, de los cuales $33.2 \%$ se realizaron con DCE, con un seguimiento promedio de 5.54 años. Se documentó mayor tasa de pérdida del injerto (HR 1.87) en los DCE comparados con los de DCS. Dentro de los factores de riesgo independientes para pérdida del injerto se identificaron: anticuerpos donante específico (DSA) positivo (HR 4.59); tiempo de isquemia de 12-24 horas (HR 2.49); tiempo isquemia más de 24 horas (HR 3.77) (2). En nuestro estudio, los tiempos de isquemia fría fueron en promedio

Tabla 2. Resultados clínicos de los receptores con DCE.

\begin{tabular}{|c|c|}
\hline Variable & $\mathrm{n}=18(\%)$ \\
\hline Función retardada del injerto & $4(22.2 \%)$ \\
\hline Falla primaria & $1(5.6 \%)$ \\
\hline \multicolumn{2}{|l|}{ TFG MDRD4 (mL/min/1.73 m²) } \\
\hline - 6 meses & 51.6 \\
\hline - 1 año & 58.4 \\
\hline - 3 años & 58.4 \\
\hline Rechazo celular al primer año & $2(11.1 \%)$ \\
\hline \multicolumn{2}{|l|}{ Supervivencia Pacientes } \\
\hline - 1 año & $88.9 \%$ \\
\hline - 3 años & $80 \%$ \\
\hline \multicolumn{2}{|l|}{ Supervivencia del injerto } \\
\hline - 1 año & $88.9 \%$ \\
\hline - 3 años & $80 \%$ \\
\hline
\end{tabular}

de 14.5 horas, lo cual pudo impactar favorablemente en los resultados.

Un estudio griego retrospectivo con 310 pacientes, 86 trasplantados con DCE y 114 con DCS, evaluó la supervivencia del injerto a uno, tres y cinco años. Comparando donante estándar con DCE, mostró que la supervivencia en este último fue significativamente inferior después del tercer año. En este estudio se categorizó a los DCE para establecer supervivencia hasta los cinco años en tipo I: donantes de más de 60 años sin factores de riesgo, tipo II: edad entre 50 y 59 años con dos o tres factores de riesgo y tipo III: mayores de 60 años con al menos un factor de riesgo. La supervivencia en el tipo I fue de $95 \%$ a uno, tres y cinco años; tipo II de $92-80 \%$ en el primer y quinto año, y en el tipo III de 78, 73 y $59 \%$ al primer, tercer y quinto año respectivamente (14). Teniendo en cuenta la clasificación anterior, nuestro estudio mostró que $38.8 \%$ de los donantes eran tipo II y III y solo $16 \%$ de tipo I. Los pacientes de nuestro estudio que fallecieron tenían más de 65 años y sus donantes eran mayores a 60 años.

Varios estudios han reportado muy buenos resultados en tasas de supervivencia del paciente y del injerto, en aquellos que recibieron trasplante de DCE, comparados con pacientes similares que continuaron en lista de espera $(6,15,16)$. Savoye muestra en su estudio, que los pacientes en lista de espera que no se trasplantan, tienen 2.3 veces más riesgo de morir que aquellos pacientes trasplantados con DCE (17). Países como España han mostrado que el promedio de edad de sus donantes es cada vez más alto, dada la disminución de muertes violentas y al aumento de donantes de mayor edad con enfermedad cerebrovascular. Batista publicó recientemente la experiencia en su centro, sin encontrar diferencias en disfunción o rechazo en aquellos pacientes trasplantados con injertos que cumplían criterios expandidos vs criterios estándar (15). Resultados similares ya habían sido descritos por Di Cocco en el 2011 (18), al igual que otros grupos como el de Remuzzi, que encontró igual supervivencia a tres y cuatro años (19). El estudio de Tennankore, comparó la supervivencia de pacientes con hemodiálisis intensiva en casa, trasplantados con donante vivo, con DCS y con DCE. Los resultados mostraron, que incluso, cuando el trasplante se realizó con DCE, la supervivencia fue mejor, al compararlos con el grupo de hemodiálisis intensiva (20).

Watson et al, encontraron que los principales predictores de pobre función del injerto fueron: edad del donante, antecedente de hipertensión arterial, obesidad, uso de adrenalina en el donante y estancia hospitalaria prolongada, hallazgos que apoyan la recomendación del uso de DCE para receptores mayores, aplicando la estrategia "old for old", que a largo plazo ha demostrado mejoría en la calidad de vida y en supervivencia del paciente al compararlo con los pacientes que continúan en la lista de espera (21-24).

Las guías españolas refieren que los riñones de DCE deben usarse preferiblemente en mayores de 60 años o en los receptores entre 55 y 60 años con factores de riesgo 
como tiempo en lista prolongado y considerarse en menores de 55 años que son hiperinmunizados (25). Si bien, existen revisiones sistemáticas que encuentran que los pacientes menores de 40 años no se benefician de recibir DCE, debe realizarse una aproximación individualizada (3). Otro grupo de pacientes en los que puede considerarse el uso de DCE, es el de aquellos con problemas de acceso vascular $(26,27)$.

En cuanto a complicaciones inmediatas, un estudio español unicéntrico observacional, comparó 60 pacientes con trasplante con DCE entre enero de 2010 a diciembre de 2012, con 14 pacientes con DCS y no encontró diferencias en supervivencia del injerto a los dos años: 95 vs $100 \%$, ni en complicaciones como función retardada del injerto, trombosis, fístula urinaria y linfocele. Sin embargo, sí hubo mayor incidencia de necrosis tubular aguda en 84.5 vs $57.1 \%(p=0.025)$ en receptores de DCE (28). Otro estudio en Latinoamérica realizado por Valjalo en Chile, mostró que 34 de 213 pacientes trasplantados renales, tuvieron DCE y presentaron más complicaciones quirúrgicas, vasculares, más rechazo agudo y hospitalizaciones y la supervivencia a cinco años fue menor en el grupo con DCE, 61 vs 89\% (29). En nuestro estudio no se presentaron complicaciones urológicas ni vasculares. La incidencia de función retardada del injerto fue $22 \%$ y la incidencia de rechazo celular al año fue $11.1 \%$, similar a lo descrito en la literatura para DCS.

En cuanto al tratamiento inmunosupresor se recomienda utilizar timoglobulina en la inducción y tacrolimus, micofenolato, esteroide como primera línea en la fase de mantenimiento (30). El 67\% de nuestros pacientes recibieron este esquema. Los esquemas libres de inhibidores de calcineurina no son recomendables en los casos de DCE, algunos autores recomiendan utilizar niveles $25-50 \%$ más bajos, con el fin de minimizar la toxicidad (13).

En nuestra población los pacientes trasplantados con DCE tienen una buena función del injerto renal a tres años con adecuada supervivencia del injerto y del paciente, similar a la reportada en pacientes trasplantados con donante estándar. Se recomienda en este grupo etáreo tener en cuenta las comorbilidades de tipo infeccioso, ya que constituyen la principal causa de mortalidad y establecer parámetros claros para el manejo de éstas.

En Colombia no existen estudios publicados de trasplante renal con DCE, por lo que socializar los desenlaces de estos pacientes puede contribuir a incentivar el uso de estos donantes.

\section{Referencias}

1. Garcia D, Ordoñez I. Guía para el manejo de la enfermedad renal crónica y Modelo de prevención y control de la enfermedad renal crónica. Basada en la evidencia. Colombia. 2007.

2. Aubert O, Kamar N, Vernerey D, Viglietti D, Martinez F, Duong-VanHuyen J-P, et al. Long term outcomes of transplantation using kidneys from expanded criteria donors: prospective, population based cohort study. BMJ. 2015;351(h3557): $1-9$

3. Pascual J, Zamora J, Pirsch JD. A Systematic Review of Kidney Transplanta- tion From Expanded. Am J Kidney Dis. 2008;52(3):553-86.

4. Merion RM. Expanded Criteria Donors for Kidney Transplantation. Transplant Proc. 2005;37:3655-7.

5. Melk A, Schmidt BM, Braun H, Vongwiwatana A, Urmson J, Zhu LF, et al. Effects of donor age and cell senescence on kidney allograft survival. Am J Transplant. 2009;9(1):114-23.

6. Audard V, Matignon M, Dahan K, Lang P, Grimbert P. Renal transplantation from extended criteria cadaveric donors: Problems and perspectives overview. Transpl Int. 2008;21(1):11-7.

7. Hwang JK, Park SC, Kwon KH, Choi BS, Kim JI, Yang CW, et al. Long-term outcomes of kidney transplantation from expanded criteria deceased donors at a single center: Comparison with standard criteria deceased donors. Transplant Proc. 2014;46(2):431-6.

8. Instituto Nacional de Salud. Informe Ejecutivo Coordinación Red Nacional de Donación y Trasplantes [Internet]. Bogotá D.C; 2018. Available from: https:// www.ins.gov.co/Direcciones/RedesSaludPublica/DonacionOrganosYTejidos/ Estadisticas/INFORME EJECUTIVO DONACIÓN Y TRASPLANTES 2018. pdf

9. Kosmoliaptsis V, Salji M, Bardsley V, Chen Y, Thiru S, Griffiths MH, et al. Baseline donor chronic renal injury confers the same transplant survival disadvantage for DCD and DBD kidneys. Am J Transplant. 2015;15(3):754-63.

10. Situación de la enfermedad renal crónica, la hipertensión arterial y la diabetes mellitus en Colombia 2017. Fondo Colombiano de Enfermedades de Alto costo, Cuenta de Alto Costo (CAC). [Internet]. Bogotá D.C; 2018. Available from: https://cuentadealtocosto.org/site/images/Publicaciones/2018/Libro_Situacion_ERC_en_Colombia_2017.pdf

11. Naghavi M, Wang H, Lozano R, Davis A, Liang X, Zhou M, et al. Global, regional, and national age-sex specific all-cause and cause-specific mortality for 240 causes of death, 1990-2013: A systematic analysis for the Global Burden of Disease Study 2013. Lancet. 2015;

12. Bozkurt B, Kiliç M. Marginal Donors in Renal Transplantation. Transplant Proc. 2015;47(5):1273-81

13. Filiopoulos V, Boletis JN. Renal transplantation with expanded criteria donors: Which is the optimal immunosuppression? World J Transplant. 2016;6(1):103.

14. Koukoulaki M, Kyriakopoulos G, Nikolaidis C, Balaska A, Pistolas D, Drakopoulos S. Proposal of categorization of expanded criteria donors in renal transplantation. Transplant Proc. 2014;46(9):3168-71.

15. Ramalho ALS, Custodio FB, Tokuda BM, Caldas HC, Fernandes IM, Abbud-Filho M, et al. Clinical and Histopathologic Comparative Analysis Between Kidney Transplant Recipients From Expanded-Criteria Donors and Standard-Criteria Donors. Transplant Proc. 2013;45(9):3234-8.

16. Flythe JE, Curhan GC, Brunelli SM. Shorter length dialysis sessions are associated with increased mortality, independent of body weight. Kidney Int. 2012;83(1):104-13.

17. Savoye E, Tamarelle D, Chalem Y, Rebibou J-M, Tuppin P. Survival benefits of kidney transplantation with expanded criteria deceased donors in patients aged 60 years and over. Transplantation. 2007;84(12):1618-24.

18. Cocco P Di, Orlando G, Rizza V, Luca L De, Angelo MD, Clemente K, et al. Kidney Transplantation From Older Donors. Transplant Proc. 2011;43(4): 1033 5 .

19.Locatelli G, Rigotti P, Baldan N, Beatini M, Valente U, Scalamogna M. Long-Term Outcome of Renal Transplantation from Older Donors. N Engl J Med. 2008;354:343-52.

20. Tennankore KK, Kim SJ, Baer HJ, Chan CT. Survival and Hospitalization for Intensive Home Hemodialysis Compared with Kidney Transplantation. $J$ Am Soc Nephrol. 2014;25:2113-20.

21.Papachristou E, Provatopoulou S, Savvidaki E, Kaplanis N, Kalliakmani P, Papasotiriou M, et al. Outcome of Transplantation in Renal Allograft Recipients From Cadaveric Donors With Standard and Expanded Criteria: A Single-Center Experience. Transplant Proc. 2014;46(9):3172-4.

22. Molnar MZ, Streja E, Kovesdy CP, Shah A, Huang E, Bunnapradist S, et al. Age and the associations of living donor and expanded criteria donor kidneys with kidney transplant outcomes. Am J Kidney Dis. 2012;59(6):841-8.

23. Pérez-Sáez MJ, Arcos E, Comas J, Crespo M, Lloveras J, Pascual J. Survival Benefit From Kidney Transplantation Using Kidneys From Deceased Donors Aged $\geq 75$ Years: A Time-Dependent Analysis. Am J Transplant. 2016;16(9):2724-33.

24. Watson JE, Johnson RJ, Birch R, Collett D, Bradley JA. A Simplified Dono Risk Index for Predicting Outcome After Deceased Donor Kidney Transplantation. Transplantation. 2012;93(3):314-8.

25.Lledó-García E, Riera L, Passas J, Paredes D, Morales JM, SánchezEscuredo A, et al. Spanish consensus document for acceptance and rejection of kidneys from expanded criteria donors. Clin Transplant. 2014;28(10):1155-66. 
26. Merion RM, Ashby VB, Wolfe RA, Distant DA, Hulbert-Shearon TE, Metzger RA, et al. Deceased-donor characteristics and the survival benefit of kidney transplantation. JAMA. 2005;294(21):2726-33.

27. Segall L, Nistor I, Pascual J, Mucsi I, Guirado L, Higgins R, et al. Criteria for and Appropriateness of Renal Transplantation in Elderly Patients With EndStage Renal Disease. Transplantation. 2016;100(10):e55-65.

28. Martínez-Vaquera S, Navarro Cabello MD, López-Andreu M, Jurado JMD,
Haad CR, Salas RO, et al. Outcomes in renal transplantation with expandedcriteria donors. Transplant Proc. 2013;45(10):3595-8.

29. Valjalo R, Reynolds E, Herrera P, Espinoza Ó, González F. Long-term outcomes with expanded criteria donors in kidney transplantation. Rev médica Chile. 2016;144(1):22-9.

30. Group KDIGO (KDIGO). TW. KDIGO clinical practice guideline for the care of kidney transplant recipients. Am J Transpl. 2009;9(Suppl 3):S1-155. 